\title{
STATISTICAL METHODS IN GENETICS
}

\author{
R. A. FISHER \\ Being the Bateson Lecture delivered at the John Innes Horticultural Institution \\ an Friday, 6th July 1951*
}

THE title chosen for this lecture is "Statistical Methods in Genetics," but after hearing what I have written you may think that it is not so much an account of the contributions which the study of Statistics has made to the advance of Genetics, as an examination of the nature of genetical Science from the point of view of a statistician. My reason for taking this point of view is that for many active years of my life I was fully engaged in doing what I could to further the progress of statistical methods, and that though for 25 years or so I have enjoyed the excitements of genetic experimentation, Genetics has only gradually come to be, for me, a whole time study, in place of a very fascinating hobby. Since that experience is in itself exceptional, it may be that the reflexions gathered on the way are worthy of record, and of interest to my genetical colleagues.

Genetics and Statistics have in common that they are both characteristic products of the twentieth century. As we should recognise them now, neither of them existed before the year 1900 . Of course I do not ignore the fact that earlier workers of many nations, Naudin and Godron, for example, Knight, Gaertner and Kohlreuter broached the practical problems of experimentation in heredity, or that on the theoretical side Galton and Weismann put forward speculations sometimes to an interesting extent near the truth. The impression upon the modern reader of these earlier workers, and it is on this criterion that I base my claim, is that the writers, intelligent as they were, did not really know what they were writing about. They had not the concepts-the framework of ideas-in terms of which their thought might have become lucid and coherent. Mendel himself should, of course, be excepted, for he was framing these very concepts. Now just the same is true, in its own field, and without a parallel exception, of the writers on Statistics of the eighteenth and nineteenth centuries. A start had been made with the problem of gathering demographic data; some beautiful mathematics, the theory of probability, had emerged in the study of games of chance; the massive intellect of Gauss had brought order into the chaotic difficulties facing astronomers and surveyors in the combination of observations.

* This lecture has been established by the John Innes Trustees to be given at the conclusion of the biennial Summer Course in memory of William Bateson. first Balfour Professor of Genetics in the University of Cambridge and first Director of the John Innes Institution, who died twenty-five years ago, 8th February i 926. 
Yet any one of the great names of the past, De Moivre or Bayes, Boole or Gauss himsclf, if by a miracle we can imagine him indoctrinated with the thought of our time, would, I believe, be astonished by the cogency and precision, the directness and accuracy with which problems formerly intolerably encumbered, can, in this age, be recognised, and resolved. In fact thcy lacked the concepts needed to think clearly about many of their problems.

Genetics and Statistics, then, have in common that each in its own field represents a distinctive point of view, which profoundly influences the intellectual processes with which scientific work is approached. I have during the past 15 years looked on, from a point of some advantage, at the process of the serologists, concerned with the human blood groups, becoming, as one may say, genotypeconscious. As this progress continues so, step by step does the worker's facility continuously sharpen in recognising the situation with which his observations confront him. What is even more striking is the confidence with which they now reach out to make new discoveries, which, without the genetic interpretation would never have been foreseen; as is clearly shown by the cases in which these advances have been indignantly repudiated as impossible, until discovery had finally and firmly established them as fact. This, indeed, is exactly what happened with the antibodies anti- $d$ and anti-e of the Rhesus blood-group system. By 1944 the existing testing fluids had been recognised to contain four different antibodies specifically related to four of the six genes by which the inheritance of this constitutional factor could be specified; for two of them however, no specific antibody was known. It was theoretically possible that no such antibodies really existed. I and others received letters vehemently insisting that no such antibodies could possibly exist, for reasons which, however, carricd no conviction, for any clear apprehension of the genetic system, revealed by tested relationships, certainly suggested that they were worth looking for. Before the end of that year indeed Mourant in England had discovered one of them, anti-e, in the serum of a patient suffering from a chronic anæmia. The important point is that he knew at once what he had discovered, and had no doubt of what tests to apply to confirm and establish his discovery. The second of the missing antibodies was more clusive; Diamond at Harvard found the first example, but in such high dilution, and with such admixtures, that it could not be used with confidence. A year or two later Hill and Haberman were able to demonstrate it unmistakeably in scra from two Louisiana negresses. The confirmation of the genetic point of view afforded by Mourant was, it may well be thought, a necessary condition for the recognition of cither the possibility or the practical importance of the second discovery to be sufficiently appreciated for it to be pursued with the confidence and tenacity needed to bring it to completion.

Only this year another antibody, this time one searched for long 
and confidently, has been added to the serologists repertoire. The system in this case is that known as $\mathrm{MN}$, first discovered in 1927 by Landsteiner and Levine using immune rabbit serum. In 1947 Race and Sanger showed that a new antibody, anti-S, in a serum from Australia subdivided each of the alleles $\mathrm{M}$ and $\mathrm{N}$ disproportionately, so that the antigen $\mathrm{S}$ with which it reacted was present in 25 out of the 53 per cent. M, but in only 8 of the 47 per cent. $N$ in the English population. Clearly the new antigen was inherited almost if not quite at the same locus as $\mathrm{M}$ and N. With the example of the Rhesus antibodies in view, it was from the first anticipated that a fourth antibody, diagnostic in this system, must also exist, complementary to anti-S, and shortly after Easter this year I learned that the long search had been rewarded. The system $M \mathcal{N} S$ s with each of four genes recognisable by

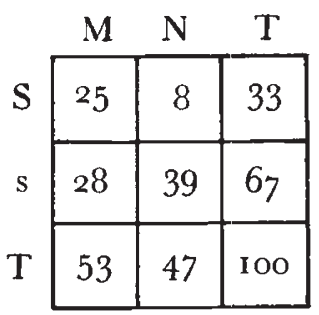

FIG. 1.-Frequencies of gene combination of the blood group factor MNSs. a separate antibody, is now the most powerful which we possess for such tasks of applied genetics as the exclusion of falsely ascribed paternity, or the recognition of interchange of the newly born in maternity hospitals.

That is only an example of the analytic penetration in biological enquiry supplied by a familiarity, not with an enormous range of genetical facts, but with primary genetical concepts, and that background which may be called the genetical point of view. A rather similar clarification must have taken place at the end of the eighteenth century with Lavoisier's formulation of chemical nomenclature. Something like it, though perhaps not so final, has occurred with the development of quantum mechanics ; but the really striking parallel of our own times is the emergence of modern statistics. Quite suddenly in the intellectual history of mankind it has become possible to think coherently and confidently about variation, a phenomenon which, when we reread nineteenth century authors, we see to have blocked and inhibited their thought to a degree which we find almost unimaginable. Apart from a universal catastrophe, we shall never think the same way again. The processes of interpretation which we apply to observational or experimental data are now capable of examination, not only rationally, but conclusively. Experimental design has become an intelligible subject for discussion, not merely by improvement in technique, but by a change in point of view. And here I may mention a connection between our two subjects which seem not to be altogether accidental, namely that the "factorial" method of experimentation, now of lively concern so far afield as the psychologists, or the industrial chemists, derives its structure and its name, from the simultaneous inheritance of Mendelian factors. Geneticists certainly need not feel that the intellectual debt is all on one side. 
A new subject for investigation will find itself opposed by indifference, by inertia, and usually by ridicule. A new point of view, however, affecting thought on a wide range of topics may expect a much fiercer antagonism. Amour propre is deeply aroused. Unproductive minds, - and God knows it is by no fault of their own that they are so--who by long occupancy of a rostrum have come to think of themselves as authorities, cannot easily brook the idea that they must reconsider their opinions. They have staked so much on their opinions being beyond question. Human nature being what it is, it is not surprising that such subjects encounter educational difficulties, and are not easily welcomed as educational disciplines, and this for the very reason which makes them educationally essential for a generation which is to live in the twentieth century, namely that they can instil the conceptional backgrounds upon which modern points of view have been established.

As the position is a serious one, and its educational importance, I believe, enormous, I do not want to give any false impression. It is not a simple matter of the jealous ignoration and exclusion of a rival field of study. That phase in a sense, has been passed. The importance of Genetics, as of Statistics, is recognised ; at least lipservice must be paid to it. At the present time there are botanical departments which like to make a show of teaching Genetics; they like it better if it can be taught as a subordinate branch of systematic Botany. There are zoologists, too, who sometimes use the language of Genetics. Some of these, however, think that it is a part of the physiology of reproduction; others that it is an aspect of experimental embryology. On these terms what they know of the subject can be accommodated to their outlook, and students anxious to learn something of Genetics can be diverted to other interests. We need not believe that such misrepresentation is consciously fraudulent. It is sufficiently explained by ignorance of what Genetics is about, and ignorance of the means that must be taken to become acquainted with the subject. Obviously, also, Genetics can and does contribute to knowledge in these other fields, but to say this is very different from saying that a study of these fields will supply, in any degree, that particular kind of understanding which it is the object of genetic studies to attain.

Statistics, it should be noted, has encountered parallel difficulties in education. The large mathematical departments, with no tradition of living contact with experimental situations, have attempted to accommodate statistical teaching as an eccentric kind of pure mathematics. Quite a number of books with statistical titles have appeared recently, especially in the United States, which show clearly the difficulty of grafting the ideas, which alone make statistics fruitful, on to a purely deductive mathematical tradition. To many, evidently, the existence of Statistics can be comfortably recognised if, and only if, it can be regarded as an application of the Theory of Probability, a 
theory which, in my innocence 30 years ago, I had thought to have been absorbed and partly superseded by the development of statistical ideas. In Paris a few years ago I was delighted to learn that the mathematicians of the Sorbonne were, with truly Gallic insight, drawing a distinction between "les probabilists" and "les statisticiens." Statistics evidently has a hard task in the atmosphere of an old mathematical department not to be changed back into something more conformable to the nineteenth century. Solid progress is indeed being made, but it is at places like Rothamsted in this country, or in the Bureau of Standards in America, rather than in places organised primarily for education.

Now, if I am right as to the inevitability of the obstacles put in the way of new disciplines, and especially of those from which we have most to gain, we can begin to appreciate the great part played by William Bateson in consolidating Genetics as an independent stream of thought, in this country, and throughout the world. We can appreciate too the wisdom of that most significant step in his career when he vacated the chair of Genetics at Cambridge, to tend and establish the nascent Institution founded by John Innes. Genetics needed a champion, and Bateson did not hesitate to recognise that he was cast for that role. Very quickly he had rejuvenated the Evolution Committee of the Royal Society, and, as the reports of that body show, had formed a group of enthusiastic workers who, in the first few years of the century, had already verified and considerably extended the Mendelian doctrine.

It is said that Bateson had no liking for statistics; and for the half-bogus statistical arguments by which that forensic adept and indefatigable controversialist, Karl Pearson, had tried to snuff out the discovery of Mendel's work, he had no reason for feeling either liking or respect. I do not think that he knew much about statistics, or that the subject seriously occupied his attention, but that he was essentially unprejudiced, the following example will show. In Igog he published his Mendel's Principles of Heredity, a book that I bought that year as a mathematical freshman. It includes a translation of Mendel's paper on "Pisum." Criticising the work on inheritance of his German predecessors, Mendel says (p. $3^{\text {I } 8) ~:-~}$

" Those who survey the work done in this department will arrive at the conviction that among all the numerous experiments made, not one has been carried out to such an extent and in such a way as to make it possible to determine the number of different forms under which the offspring of hybrids appear, or to arrange these forms with certainty according to their separate generations, or definitely to ascertain their statistical relations."

Bateson takes the opportunity to add the following significant footnote :-

"It is to the clear conception of these three primary necessities 
that the whole success of Mendel's work is due. So far as I know this conception was absolutely new in his day."

In rgog Bateson had won his campaign, on the scientific front. On the academic front he had perhaps reconnoitred the position. Whatever may have bcen his reflections, it is clear that they led him to conclude that it was in the Rescarch Institution rather than in the University Department that Genetics could grow to its full stature. He may have felt that he himself had something better to do than to compete, in an atmosphere of rather feline jealousy, with some one or other somewhat decayed branch of traditional Zoology.

I do not know how much foresight to ascribe to Bateson in this matter. Looking back it is, I think, clear to all of us that his choice was wise, and in itself constituted a major contribution to the progress of Genetics. Obviously, the conditions for teaching the subject in Cambridge would now have been better if Bateson had sacrificed the best years of his life, all too short as it proved to be, to grappling with the obstacles, which would have been thrown in his way. In principle, at least, I am glad that the John Innes bequest offered an alternative to such a sacrifice.

One great advantage of Genetics over the earlier biological studies is that it brings the research worker into close contact with living material. It should occupy his attention, and be under his constant supervision. As Bateson so often insisted the gencticists laboratory is the garden plot, or the breeding pen. It is not, I believe, sufficiently realised that this need for absolute realism is particularly required in statistical work when applied to genetic purposes. It is in general the statisticians task to bring theory into a truly organic coherence with objective and verifiable observations. Genetics is almost alone among the biological sciences in having a definite and compact, though doubtless imperfect, theoretical basis. Its characteristic frequencies are a constant stimulus to statistical enquiry. An important step was taken towards making Statistics competent to aid in Genetics, when the theory of small samples was developed. So long as the statistician was supposed to concern himself only with vast aggregates of massed data, his acquaintance with the detailed processes by which they came into existence was bound to be vague ; each part of the whole contained its own complications and its own enigmas ; in the treatment of the mass these were necessarily almost wholly unrecognisable, and the interpretation was harassed by innumerable unanswerable queries. As soon, however, as it was realised that small bodies of data, such as the progeny of a single mating, could be examined by competent and exact methods, the particular circumstances of each case could be considered, the homogeneity of different groups examined, and generally speaking, a check could be put to the process by which good data are contaminated by bad.

Direct contact with what is actually done in experimentation 
helps the statistician in another very essential way, by leading him to consider variations in procedure, and the reasons why one method is to be preferred to others. The whole wide subject of experimental design is opened out by this consideration. Genetics is indeed in a peculiarly favoured condition in that Providence has shielded the geneticist from many of the difficulties of a reliably controlled comparison. The different genotypes possible from the same mating have been beautifully randomised by the meiotic process. A more perfect control of conditions is scarcely possible, than that of different genotypes appearing in the same litter. Generally speaking the geneticist, even if he foolishly wanted to, could not introduce systematic errors into the comparison of genotypes, because for most of the relevant time he has not yet recognised them. What a beautifully controlled experiment, again, is put into the geneticist's hands in a linkage test by simple backcross to a multiple recessive. Here each pair of allelomorphic genes should occur, though in different combinations, equally in each of the pairs of complementary genotypes which constitute his results. The first step to perfect control against viability disturbances, is then inherent in his material.

Spoiled favourites of the gods, as in this matter they are, geneticists have been in some cases a little slow to make use of experimental precautions, which in other fields have been found to be necessary. Some years ago, in the early days of quantitative studies, it was quite usual for an investigator of a wide varietal cross to provide data showing that the $F_{2}$ was more variable than the $F_{1}$. Of course, this was very probaibly true; and if true it was certainly of genetic significance. But an $F_{1}$ of perhaps $5^{\circ}$ plants carefully grown on a small uniform plot is not cursorily to be compared with an $F_{2}$ of 5000 , set out on a hundred times the area. The geneticists of those days, however, showed no awareness that even clonal material, compared in this way would probably have shown some difference in variability in the direction observed. Their humble colleagues in farm institutes had no doubt heard of soil heterogeneity, and the more intelligent of them could perhaps have suggested how an unbiassed comparison could have been made, but, at that time, such knowledge was not expected of geneticists. Indeed to this day, I would not know where to turn up, in an elementary textbook of Genetics, where surely it belongs, an account of how such a comparison could properly be made.

That example, although exceedingly simple, may be said to belong to the field of biometrical Genetics, where statistical ideas are admittedly essential. In linkage studies, however, the same point may be made. I mentioned further back how well controlled a linkage backcross was from disturbances due to unequal viability. Well controlled, but not perfectly controlled, for differential viability due to interaction of two or more factors will still, in a single cross, disturb the recombination fractions. Here the gods have provided 
the remedy, in that the test can be carried out in two ways, in coupling, or in repulsion. Using both tests the effects of differential viability can be measured and eliminated. In three-point tests, moreover, where four modes of gamete formation can be recognised, there are four types of triple heterozygote available; and in four-point tests

Mode of gamete formation.

\begin{tabular}{c|c|c|c|c|}
\cline { 2 - 4 } $\begin{array}{c}\text { Genotye } \\
\text { of parent }\end{array}$ & None $(\mathrm{aA})$ & $(\mathrm{bB})$ & $(\mathrm{cC})$ \\
\hline$+/ \mathrm{abc}$ & $\mathrm{P}$ & $\mathrm{Q}$ & $\mathrm{R}$ & $\mathrm{S}$ \\
\hline $\mathrm{aBC} / \mathrm{Abc}$ & $\mathrm{Q}$ & $\mathrm{P}$ & $\mathrm{S}$ & $\mathrm{R}$ \\
\hline $\mathrm{AbC} / \mathrm{aBc}$ & $\mathrm{R}$ & $\mathrm{S}$ & $\mathrm{P}$ & $\mathrm{Q}$ \\
\hline $\mathrm{ABc} / \mathrm{abC}$ & $\mathrm{S}$ & $\mathrm{R}$ & $\mathrm{Q}$ & $\mathrm{P}$ \\
\hline
\end{tabular}

Fig. 2.-Complete three-point test using all four triple heterozygotes. Column headings show which, if any, gene pairs are interchanged. Four pairs of genotypes of offspring are represented by the letters $P, Q, R, S$. there are eight. By no merit of ours, indeed, the means are always available for making a clean evaluation. But very seldom have the means been used. In the literature of Drosophila, I have found a few cases of a complete three-point test. They are very bad. I doubt if a four-point test has ever been made in all eight ways. In the examples I have found, indeed, the purpose of the procedure has been gravely misunderstood, and the counts merely addcd up, in the naïve expectation that $a x+b y$ is equivalent to $a y+b x$ ! Admirable as the early work with Drosophila was, the leading drosophilists of the Unitcd

States were quite singularly unaware that anything was to be learned from statistical methods. Some seem to have cultivated the exceedingly misleading doctrine that with large enough numbers there was no need for critical experimentation.

I mentioned just now the numbers of modes of gamete formation in a multiple backcross and the numbers, which happened to be the same, of multiple heterozygotes which could be used for breeding. What I said was obvious enough to those familiar to this type of work, yet I find that students are often surprised at finding that Genetics requires a recognition of combinatorial relationships. Yet from Mendel's paper to our own day, the primary distinctions of the language of geneticists have been combinatorial. In using terms like homozygote and heterozygote we are intuitively recognising that the number 2 may be made up of like parts, or of unlike parts. I believe this combinatorial aspect can usefully be brought to the attention of students, and as soon as the theory of polysomic inheritance is touched on it becomes a sheet anchor. In tetrasomic organisms instead of two kinds of organism defined by the partition of the number 2, we have five kinds defined by the partitions of the number 4, and in hexasomics there are II, only one of which is homogenic, corresponding with the homozygote, while ro correspond, but in considerably different ways, to the heterozygote, and contain various numbers of different allelomorphs.

In linkage studies with two loci disomic organisms present only 
two doubly heterozygous types, distinguished as in coupling and in repulsion, which exhibit the effects of linkage in their gametic series. These two types are equivalent, or isomorphic, in their behaviour, since the gametic series of one is transformed into that of the other by a mere transposition of classes. With two factors in an autotetraploid the number of genotypes capable of exhibiting linkage effects is not two but no less than two hundred and sixty-six, but these may be recognised to fall into only twenty-four isomorphic sets, each capable of supplying its own peculiar information as to the linkage situation. The recognition of these sets is thus the first step to classifying an otherwise chaotic mass of possibilities. The results of using plants of any one set require to be supplemented by the results of using others.

In disomic work with two factors, there are only two modes of gamete formation which can be distinguished, the recombinants and the non-recombinants. With tetrasomic inheritance eleven modes are distinguishable. The frequencies with which these occur must be determined experimentally. When their values are more familiar, we may be able to recognise on what features of the meiotic process, and of the positions of the loci, they depend. Three features are easily recognisable, the frequency of recombinant strands, and the two frequencies of "double reduction," characteristic of the two loci. The observable frequencies do not specify the frequency of quadrivalent formation, nor enable us to compare exactly the frequencies of recombination in bivalents and quadrivalents. They do, however, supply an indication of the position of the centromere relative to the loci marked. Speaking of the future, the existence of eleven distinct modes of gamete formation, having frequencies capable of evaluation by direct breeding experiments, will certainly throw light from a new aspect on the geometry and mechanics of the meiotic process, apart from their primary naturalistic function of predicting the gametic output.

The experimental determination of these frequencies is not easy. Whereas in disomic material a simple backcross to a multiple recessive will serve to determine the genotype of each gamete that has been effective, the corresponding diagnosis of the diploid gametes of a tetraploid, or of the triploid gametes of a hexaploid requires successive backcrosses in two generations. Such work is relatively costly in space and time, but not extravagantly so. I grow every year rather more plants than did Gregor Mendel, but not three times as many. I am an advocate of economy in experimentation, but not of the cramping dogma that everything should be done with a few milk bottles, or petri-dishes.

A circumstance much more baffling than the need for a double backcross comes into view when we observe that having determined the genotypes of a sample of gametes, we have not thereby determined the mode of gamete formation by which each arose. Not only does 
each mode of gamete formation give rise to several different genotypes, just as in disomic forms complementary pairs of genotypes have a similar origin, but, at the same time, the same genotype of gametes may be produced in more than one way. The relationship between the genotypes of the gametes formed and their possible modes of formation, is somewhat more complex than that to which we are used in disomic inheritance. In place of a simple series, we have an array of series, or what mathematicians call a matrix. The relation between the modes of gamete formation and the genotypes to which they give rise is established by an essentially simple, though sometimes tedious operation known as matrix multiplication. Now, let me not be

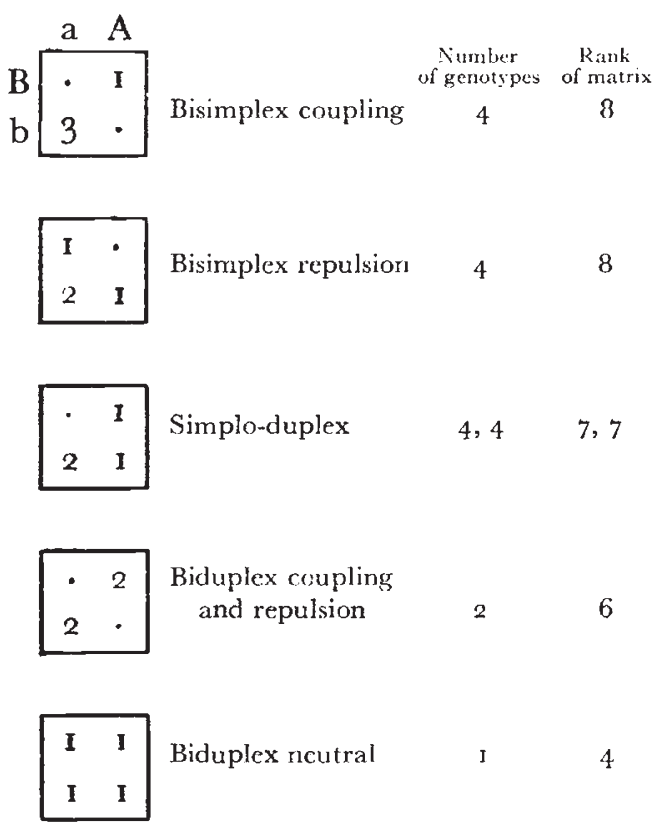

FrG. 3.-Six isomorphic sets of doubly heterogenic genotypes.

accused of introducing matrix algebra into Genetics, by a wanton act of highbrow sophistication. Rather consider Lythrum salicaria as making me recognise that its genetic operations may be compendiously mapped only by setting out the 24 gametic matrices corresponding with its 24 sets of isomorphic genotypes.

Of these 24 only 6 are available unless more than two alleles are available at the same locus. The simplest and most fundamental properties of these matrices are naturally of direct genetic concern. For example the rank of each matrix tells us the number of independent equations which experimentation can supply towards determining the eleven unknown frequencies of the modes of gamete formation. One has rank 4, one has rank 6, two have rank 7 and two rank 8. Each set of genotypes by itself can only supply partial information. Hence 
the need for experimentation with genotypes of more than one isomorphic set. By combining data from two different sets I have brought the rank up to 9 . With a third set I hope to bring it up to ro. The one piece of information that remains is inaccessible, unless three alleles can be mobilised at both of the two linked loci.

Now, I have retailed these details from my own work merely to underline and emphasise a general fact relevant to the present status of Genetics, namely that we shall not understand the process of inheritance as it occurs in nature unless we follow Mendel's example, and endeavour as he says " to determine the number of different forms under which the offspring of hybrids appear," and to that end extend, so far as our material requires it, those simpler combinatorial concepts which Mendel himself applied to the factorial system of unlinked factors in diploids. Of course, one can understand the academic mind seeking for problems of Ph.D. magnitude, and for material making the most modest demands upon space or time. After all the purpose of the Ph.D. thesis is to exhibit such competence as should be acquired in a successful apprenticeship. The limitations of relevance, and the monotony of ideas are proverbial. One does not want to go on doing that sort of thing all one's life. Moreover, the advancement of our science demands that we should not. Mansize problems are not hard to find. From the theoretical point of view it would be intolerable if we were unable to understand the process of inheritance in, for example, the cultivated potato; while on the applied side the art of potato breeding will owe little to genetical science so long as geneticists have no experience of following segregation in that species, of investigating the peculiar genetical situations it presents, or of carrying out manipulative breeding projects with this difficult material.

The potential value of Genetics to the human race, lies in the enormous variety of genotypes which can be compounded by the combination of relatively few, though absolutely numerous, elements. In this it is in a position strikingly similar to that of Chemistry. Our elements are genes, and our compounds genotypes. The extension of interest to polysomic inheritance is in this analogy parallel to the extension which took place in Chemistry when the so-called " organic" compounds with their covalent bonds began to occupy attention, alongside the older Chemistry of acids and bases. Organic Chemistry looked at first intolerably complicated, and many of the older simplifications broke down. Yet its study, however discouraging at first, has certainly been rewarding, and I do not doubt that in a later generation experts in the manipulation of polysomic forms will be astonished at our lack of curiosity in exploring the new field, and at our lack of enterprise in exploiting it.

For the future of Genetics we may, I think, gain some hints from the way Chemistry has developed, for as a science with an effective theoretical basis it has a start of about a century over Genetics. In 
that time the chemists have been able to provide themselves with a great variety of reagents, purified materials the reactions of which are largely known. Without them the chemist would be unable to recognise the chemical entities with which he has to deal, to examine what goes on in a chemical process, or to synthesise desired compounds. The geneticist has exactly parallel needs. We are aware of the need for multiple recessives, and for inbred lines as tools with which to probe an obscure genetical situation, but in the present state of our science we usually have to set about making the tools before we can use them. We do not find much already made. In examining the fifth linkage group of the house-mouse I spent 9 generations in making up a quadruple recessive between closely linked factors. This was not in the least extravagant, but, on the contrary, a very profitable investment. Such preparation work, involving a programme of preliminary matings, should be more general, and should occupy a larger proportion of a department's resources than is generally recognised. In a teaching department, to have such work going on is a first necessity, if anything like instruction in practical Genetics is to be attempted, for the preparation of genetic material needs both understanding and experience. I should suggest, too, that the continuity of policy which alone is possible at a great Institution devoted to genetic research, the routine analysis which is perfectly suitably carried out by trainees should be supplemented and supported by a permanent policy of preserving and enriching the collection of available genes and of reliably true breeding strains, the reactions of which are well known from previous experience.

Well that perhaps is as much as I can hope to put into an hour's discourse. We are the heirs of a tradition of potent ideas. The future of our science is full of promise. The more we reflect on the living creation with these ideas in mind the more there appears to be that can hopefully and fruitfully be attempted. Every generation in our science has the task of developing more penetrating and effective breeding techniques, for investigation, and for development. The full promise of our growing science can only be realised as we study these techniques, and apply them to those many problems which will only yield to professionally trained skill and experience. To preserve every point gained, and to enrich our armoury of resources, is especially the task of the permanent research establishments ; and it is for his foresight in accepting the directorship of John Innes' foundation that geneticists will always honour the name of William Bateson. 